\title{
FOREWORD
}

\section{SPECIAL ISSUE ON STIMULUS EQUIVALENCE}

\author{
LANNY FIELDS \\ The College of Staten Island \\ and the \\ Graduate Center of the City University of New York
}

What are equivalence classes? How are they related to other conceptual categories? Why the recent interest in the study of equivalence classes? Finally, why is this Special Issue of The Psychological Record devoted to the topic?

When many stimuli that resemble one another come to evoke similar responses in the absence of direct training, they are said to act as members of a class. The class may be considered to be classical, relational, probabilistic, or fuzzy depending on the formalism used to measure the physical similarity of the stimuli. Much has been learned about the experimental conditions needed to establish such classes.

Alternatively, stimuli that do not resemble each other can also come to be related. Such stimuli constitute equivalence classes. An example would be the picture of a dog, the words DOG, POOCH, CHIEN, and PERRO as written, and the sound of each word. In a set of $\mathrm{N}$ such stimuli, there are $\mathrm{N}^{2}$ potential pair-wise relations. Only some of those relations have to be established by training. If, after learning those relations, a student (or subject) recognizes the relatedness of the stimuli in all remaining untrained relations, the stimuli are said to function as an equivalence class. In addition, once a set of stimuli becomes an equivalence class, a response trained to one class member will transfer to the other members of the class. This too occurs without benefit of direct training. To illustrate, after a young child in a multicultural society has learned the relations DOG-POOCH, POOCH-CHIEN, and CHIENPERRO, the child should spontaneously and immediately recognize that PERRO is related to DOG and POOCH. In addition, when taught to say "WOOF" in the presence of the word PERRO, the child would make that sound in the presence of the other words in the class.

If one can reliably form equivalence classes, one can reliably produce novel and appropriate performances in the presence of perceptually dissimilar events. Language related phenomena such as referentially based meaning, comprehension, syntax, crossmodal perception, semantic memory, and the transfer of expressive repertoires are characterized by such performances. Understanding how equivalence classes are established, then, could shed light on the 
development of these linguistic phenomena which are taken to indicate the operation of higher-order cognitive processes. It is this potential for explaining many aspects of language that has raised much interest in the study of equivalence classes among the members of the behavior analytic community.

Historically, principles of mediation have been invoked to account for the linkage of stimuli in an equivalence class. The experimental conditions that could reliably induce such classes, however, were not clearly understood. Research that focused on the identification of the conditions that influence the formation of equivalence classes began in 1971. This approach involved the study of single subjects and the direct measurement of test performances that were indicative of class formation. The pace of research was slow for about ten years. Thereafter, the publication rate accelerated markedly, yielding a substantial increase in the range of topics being explored, and the inevitable emergence of theoretical controversies.

In addition, a perusal of the research literature for the last twenty years suggested that there were groups of researchers who were working in areas that are equivalent to equivalence. These researchers were using different terminology to consider the same or closely related phenomena, and the same terminology to denote different phenomena. Also, the theoretical assumptions held by one group were not cited by others. The same literature review also made it apparent that work on equivalence or equivalence-like processes was being conducted with human and nonhuman subjects; once again, there was relatively little impact across research domains.

The time seemed ripe for a gathering of researchers in these somewhat isolated domains to present and discuss their current work in a setting that would foster critical discussion and debate. Would the work being conducted with one species be informative to those doing similar research with other species? Would a cross-species perspective provide a more comprehensive and systematic understanding of the formation of equivalence classes? Would equivalences between disparate, but formally similar, terminology emerge? Would data and ideas held by one group provide new insights to members of other groups?

A natural venue for such a meeting was the Annual Symposium for the Quantatitive Analyses of Behavior. The topic for the 1992 meeting was chosen to be stimulus relations. The meeting was held on June 1214, 1992 in James Hall at Harvard University, and was sponsored by the Society for Quantitative Analyses of Behavior, the Department of Psychology of Harvard University, The College of Staten Island of the City University of New York, the Cambridge Center for Behavioral Studies, and the Program in Psychiatry and Law of the Harvard Medical School. We invited representatives from most of the laboratories studying stimulus equivalence with human subjects and other representatives from laboratories studying concept formation, categorization, or language development with nonhuman subjects. The 
presenters came from the United States, Great Britain, and Ireland. Twenty five papers were presented over a three-day period.

The scope of the papers was broad and the depth of each presentation was noteworthy. Many of the hoped-for interactions emerged. We thus felt that it would be desirable to find a means of publishing a refined version of many of these papers. The Psychological Record was interested in the project; this Special Issue is the product of that endeavor. Tony Nevin and I acted as the Editors for this issue. Each article underwent peer review before acceptance for publication. The eighteen articles in this special issue are representative of the content of the Symposium itself.

Articles in this volume are grouped thematically into four clusters. Articles in the first cluster consider some historical roots of equivalence and the linkage of equivalence classes to naturally occurring categories and cross-modal perception. Articles in the second cluster consider the relation of equivalence classes to complex cognitive function such as inferential transitivity, syntax, language comprehension, linguistic expression, neural-network connectionist models, decision making in complex medical settings, and the development of drug addiction. Articles in the third cluster consider how the structure of an equivalence class influences test performances and also determines the relatedness of the stimuli in such a class. Articles in the fourth cluster consider how functional classes and equivalence classes are formed by nonhumans. The data described in these articles demonstrate cognitive processes in nonhumans and also have important implications for theories of language development. Although an article is placed within a cluster, in many cases, articles in different clusters make contact with similar issues.

We hope this volume will provide a comprehensive introduction to the topic of stimulus equivalence for the reader who is unfamiliar with this exciting and fruitful area of research. We also hope the articles provide a broad ranging summary of state-of-the-art study of stimulus equivalence as of 1992. 